\title{
Estudio Computacional de la Cinética y Mecanismos de Reducción del Colorante Rojo Allura por Bisulfito de Sodio en Fase Acuosa
}

\author{
Adolfo E. Ensuncho, Jesús M. López y Juana Robles \\ Universidad de Córdoba, Departamento de Química, Grupo de Química Computacional, Cra 6 № 74-103, \\ Córdoba-Colombia (e-mail: adem9abril@hotmail.com; jmanuellopez.gqc@gmail.com, \\ juanarobles2003@yahoo.com)
}

Recibido Ago. 22, 2012; Aceptado Oct. 16, 2012; Versión final recibida Dic. 16, 2012

\begin{abstract}
Resumen
Se determinó la cinética de la reducción del colorante rojo allura (E-129, C.I. 16035) por bisulfito de sodio al nivel B3LYP/6-31G(d) en solución acuosa. Esto con el fin de evaluar una ruta mecanística plausible para la reacción de reducción de este importante azo colorante usado en la industria de alimentos. Las constantes de velocidad predichas para la etapa de sulfonación $\left(\mathrm{k}_{\text {sulf }}\right)$ estuvieron en buen acuerdo con respecto a las constantes de velocidad experimentales reportadas para algunos colorantes de estructura química similar a rojo allura. Se localizaron cinco puntos estacionarios sobre la superficie de energía potencial B3LYP. Los estados de transición para la primera y segunda etapa de la reacción de reducción son presentados por primera vez y caracterizados mediante cálculos de la coordenada intrínseca de reacción y frecuencias vibracionales. Los parámetros termodinámicos de activación indicaron que la reacción fue estable, endotérmica y no espontánea.
\end{abstract}

Palabras clave: bisulfito de sodio, rojo allura, reacción de reducción, cálculos DFT, azo colorantes

\section{Computational Study of the Kinetics and Mechanism of the Reduction of Allura Red Dye by Sodium Bisulfite in Aqueous Phase}

\begin{abstract}
The reduction kinetics of allura red dye (E-129, C.I. 16035) by sodium bisulfite at level B3LYP/6-31G(d) in aqueous phase was determined. This was done to evaluate a plausible mechanistic path for the reduction reaction of this important azo dye used in the food industry. The predicted rate constants $\left(k_{\text {sulf }}\right)$ for the sulfonation step were in good agreement with respect to experimental rate constants reported for some dyes of chemical structure similar to Allura red. On the B3LYP potential energy surface, were located five stationary points corresponding to reactants, transition states and products. The transition states for the first and second step of the reduction reaction are presented for the first time and characterized by calculations of intrinsic reaction coordinate and vibrational frequencies. The activation thermodynamic parameters indicated than the reaction was stable, endothermic and non-spontaneous.
\end{abstract}




\section{INTRODUCCIÓN}

El colorante azoico rojo allura (E-129, C.I. 16035) es usado como aditivo confiable en una amplia variedad de productos alimenticios con el fin de mejorar sus características visuales y así atraer las preferencias de los consumidores (Fallico et al., 2011). Fue admitido por la FDA (Food and Drug Administration) para su uso en la industria alimentaria de los Estados Unidos a mediados de la década de los ochenta para reemplazar el colorante amaranto (E-123, C.I. 16185), ya que este fue prohibido por mostrar efectos nocivos en la salud (Wilson y Bahna, 2005; Umer et al., 2008; Holmberg, 1978). Se ha encontrado que, el uso de estos colorantes en bebidas suaves (jugos de frutas) se ha limitado por su sensibilidad a sufrir decoloraciones que pueden ocurrir en presencia del conservante bisulfito de sodio, el cual participa en este proceso mediante adición nucleofílica (Scotter y Castle, 2004; Gemeay, 2002). Este hecho fue demostrado por Damant y colaboradores, quienes mediante estudios de espectrometría de masas y cromatografía líquida de alta resolución (HPLC, por sus siglas en inglés), indicaron que el colorante amarillo anaranjado (E-110, C.I.15985) en presencia del ión bisulfito formaba un compuesto coloreado amarillo-limón (Damant et al.,1989). Asimismo, por subsecuentes estudios de resonancia magnética nuclear (RMN, por sus siglas en inglés) y espectrometría de masas se mostró que la adición nucleofílica del ión bisulfito ocurría sobre el carbono en la posición 4 de la molécula de amarillo anaranjado (Damant et al.,1989).

Otros trabajos que indican que la posición 4 se ve favorecida para la adición nucleofílica del bisulfito han sido reportados (Berke et al.,1998). También, mediante el uso de descriptores de la reactividad locales en el contexto conceptual de la teoría del funcional de la densidad (DFT, por sus siglas en inglés) se mostró que el sitio preferido de ataque del agente reductor correspondía al carbono en la posición 4, para amarillo anaranjado y rojo allura, ya que sobre este sitio en la molécula se obtuvo el valor más alto de la función de Fukui para el ataque nucleofílico (Ensuncho et al., 2012). Recientemente, algunos estudios cinéticos y mecanísticos empleando técnicas espectrofotométricas convencionales, arrojaron resultados similares a los ya mencionados (Gemeay, 2002; Berke et al., 1998; Salem y Gemeay, 2000). A pesar de la importancia de estas reacciones en la industria de alimentos, la literatura revela pocos estudios al respecto (Ozen et al., 2003; 2004).

Recientemente, Gemeay determinó la cinética de reducción del colorante amarillo anaranjado por bisulfito de sodio, manteniendo constante la temperatura y concentración del colorante, pero variando la concentración de bisulfito (Gemeay, 2002). En el estudio, se encontró que el bisulfito de sodio, a concentraciones menores de $0.04 \mathrm{M}$, la reducción del colorante no ocurría. Además, la presencia de puntos isosbésticos en los espectros de absorción UV/Vis obtenidos, mostraron claramente la reversibilidad de esta reacción en condiciones usuales (como es el caso de la industria de alimentos donde el jugo de frutas no contiene un exceso de bisulfito). Asimismo, el mecanismo de reacción para la reducción de amarillo anaranjado por bisulfito fue planteado en dos etapas. La primera correspondió a la interconversión entre los tautómeros azo e hidrazona del colorante mediante una reacción de transferencia de protón intramolecular (Zimmerman y Wang, 2002; Beyramabadi y Morsali, 2011) y la segunda etapa, consistió en la adición nucleofílica de bisulfito sobre la especie hidrazona en la posición 4 de la molécula de colorante, produciendo el correspondiente aducto sulfonado (Gemeay, 2002). Puesto que la evidencia experimental es concluyente sobre la existencia de los equilibrios entre el colorante amarillo anaranjado y los aductos sulfonados, se propuso el estudio computacional de la cinética de reducción del colorante rojo allura, el cual presenta una estructura química similar a la de amarillo anaranjado, teniendo en cuenta las dos etapas de la reacción. Además es de nuestro conocimiento, que para el colorante rojo allura no hay estudios ni experimentales ni computacionales de su cinética de reducción frente a bisulfito de sodio. Por ello, el presente trabajo se enfoca en el estudio cinético y mecanístico de este colorante desde el ámbito computacional, basándose fundamentalmente en la ruta mecanística reportada para el colorante amarillo anaranjado por Gemeay (Gemeay, 2002). Se espera que el estudio contribuya a la comprensión del fenómeno de degradación del color en azo colorantes desde un punto de vista atómico y molecular.

\section{METODOLOGÍA}

\section{Detalles Computacionales}

Todos los cálculos computacionales se llevaron a cabo usando el funcional híbrido B3LYP (Ruangpornvisuti y Wanno, 2004; Du et al., 2004; Lee et al., 1988), implementado en el paquete Gaussian03 (Rev. E.01) bajo plataforma Linux (Frisch, 2004). Las optimizaciones geométricas para los diferentes puntos estacionarios (reactivos, estados de transición y productos) sobre la superficie de energía potencial de la reacción de reducción del colorante rojo allura, se desarrollaron con el conjunto de funciones base 6-31G(d) (Del Bene y Shavitt, 1994; Saebo y Radom, 1983). Las estructuras de los estados de transición para las etapas de tautomerización y sulfonación (designados como $\mathrm{TS}^{\ddagger T a u t}$ y $\mathrm{TS}^{\ddagger T S u l f}$ ) respectivamente, se localizaron usando el método QST2 (Rincón y Toro-Labbé, 2007), el cual emplea la aproximación de tránsito sincrónico 
cuadrático para acercarse a la región cuadrática del estado de transición y posteriormente a través del algoritmo cuasi-Newton se finaliza la optimización. Además, se llevaron a cabo cálculos de frecuencias para verificar la naturaleza de los puntos estacionarios localizados sobre la superficie de energía potencial para la reacción de reducción, como mínimos o estados de transición de primer orden al presentar solo una frecuencia imaginaria. Las energías totales calculadas al nivel B3LYP fueron corregidas con un factor de escala de 0.980 para las energías del punto cero (Lewars, 2010; Foresman y Frisch, 1996). La energía del punto cero, es una corrección a la energía electrónica de la molécula, para dar cuenta que a cero Kelvin (0k), las vibraciones moleculares aun persisten, lo cual es una consecuencia directa del principio de incertidumbre (Levine et al., 2008). Las constantes de velocidad (k), fueron calculadas con base al formalismo propuesto por Eyring (Rooney, 1995; Jensen, 2006) en el marco de la teoría clásica del estado de transición (ecuación 1).

$k=\frac{k_{B} T}{h} e^{-\frac{\Delta G^{\ddagger}}{R T}}$

Donde $k_{\mathrm{B}}$ es la constante de Boltzmann, $T$ es la temperatura absoluta, h la constante de Planck y $\Delta G^{\ddagger}$ es la energía Gibbs de activación para la reacción de reducción del colorante. Todos los cálculos de frecuencias que se llevan a cabo en el programa Gaussian (Frisch, 2004), incluyen un análisis termoquímico del sistema (Ochterski, 2000). Por lo tanto, mediante este programa se predicen importantes parámetros termodinámicos a temperatura y presión específica, tales como; la corrección térmica a la energía, la capacidad calorífica y entropía. Las temperaturas que se fijaron para realizar el análisis termoquímico fueron 298.15K, 303.15K, 308.15K, 313.15K y 318.15K respectivamente. Ahora bien, para establecer las diferentes cantidades termodinámicas calculadas en este trabajo, se usaron las siguientes ecuaciones:

$\mathrm{E}_{0}=\mathrm{E}_{\text {elec }}+\mathrm{ZPE}$

Donde $E_{0}$ es la energía térmica interna corregida, $E_{\text {elec }}$ es la energía electrónica y ZPE, corresponde a la energía del punto cero. En la ecuación 3, E corresponde a la energía total, debida a las contribuciones electrónica, rotacional, vibracional y translacional.

$\mathrm{E}=\mathrm{E}_{0}+\mathrm{E}_{\mathrm{rot}}+\mathrm{E}_{\mathrm{vib}}+\mathrm{E}_{\text {tras }}$

Ahora bien, la entalpía $(H)$ y energía libre de Gibss $(G)$ se pueden obtener mediante las ecuaciones 4 y 5 .

$$
\begin{aligned}
& H=E+R T \\
& G=H+T S
\end{aligned}
$$

De las ecuaciones 4 y $5, \mathrm{R}$ corresponde a la constante universal de los gases, $\mathrm{T}$ es la temperatura absoluta y $\mathrm{S}$ corresponde a la entropía. Por otro lado se debe tener presente que, las cantidades termodinámicas calculadas anteriormente, tienen como punto de partida la función de partición q(V,T) (McQuarrie 2000). Así, la función de partición para cualquier componente translacional, vibracional, rotacional o electrónico, se puede determinar usando la ecuación 6.

$$
\mathrm{S}=\mathrm{Nk}_{\mathrm{B}}+\mathrm{Nk_{B }} \ln \left(\frac{\mathrm{q}(\mathrm{V}, \mathrm{T})}{\mathrm{N}}\right)+\mathrm{Nk}_{\mathrm{B}} \mathrm{T}\left(\frac{\partial \mathrm{lnq}}{\partial \mathrm{T}}\right)_{\mathrm{V}}
$$

Aunque la forma usada en el programa Gaussian es un tanto especial (Ochterski, 2000) y la ecuación 6, toma la forma de la ecuación 7.

$$
S=R\left(\ln \left(q_{t} q_{e} q_{r} q_{v} e\right)+\mathrm{T}\left(\frac{\partial \ln q}{\partial \mathrm{T}}\right)_{V}\right.
$$

La energía térmica interna E, puede además ser obtenida a partir de la función de partición (ecuación 8).

$$
E=N_{B} T^{2}\left(\frac{\partial \operatorname{lnq}}{\partial T}\right)_{V}
$$

Finalmente, la energía calculada mediante la ecuación 8, se puede usar para obtener la capacidad calorífica (ecuación 9). 


$$
\mathrm{C}_{\mathrm{v}}=\left(\frac{\partial \mathrm{E}}{\partial \mathrm{T}}\right)_{\mathrm{N}, \mathrm{V}}
$$

Las ecuaciones 7, 8 y 9, se usan para derivar las expresiones finales para calcular los diferentes componentes de las cantidades termodinámicas arrojadas por Gaussian 03. En cuanto a los parámetros termodinámicos de activación $\Delta \mathrm{G}^{\ddagger}$ (energía de Gibbs de activación), $\Delta \mathrm{H}^{\ddagger}$ (entalpía de activación) y $\Delta \mathrm{S}^{\ddagger}$ (entropía de activación), como es usual fueron calculados como la diferencia de estas cantidades para reactivos y estado de transición, como se muestra en las ecuaciones 10,11 y 12 respectivamente.

$$
\begin{aligned}
\Delta G^{\ddagger} & =G^{T S}-\sum G^{R} \\
\Delta H^{\ddagger} & =H^{T S}-\sum H^{R} \\
\Delta S^{\ddagger} & =S^{T S}-\sum S^{R}
\end{aligned}
$$

De las ecuaciones 10, 11 y 12, los exponentes TS y $R$, indican estado de transición y reactivos respectivamente. La vía de reacción fue seguida por el método de coordenada intrínseca de reacción (IRC, por sus siglas en inglés) (Yamashita et al., 1981; Wang et al., 2006) con un tamaño de paso $n=10$ en unidades de 0.1 u.m. $a^{-0.5}$ bohr y número de pasos $\mathrm{N}=20$ a partir de la geometría del estado de transición conectando dos mínimos. El modelo de solvente del continuo polarizado (PCM, por sus siglas en inglés), fue empleado para considerar los efectos del solvente sobre la reacción (Tomasi et al., 2005), usando una constante dieléctrica $(\varepsilon)$ de 78.5 para la fase acuosa.

\section{RESULTADOS Y DISCUSIÓN}

En la figura 1, se presenta la vía de reacción investigada en este trabajo. Se localizaron cinco puntos estacionarios, correspondientes a reactivos, estados de transición y productos respectivamente. Los reactivos se caracterizaron como mínimos al no presentar valores negativos de la matriz Hessiana, mientras que los estados de transición fueron caracterizados con frecuencias imaginarias de -1278.97i para TS ${ }^{\ddagger T a u t}$ y -566.811i para $\mathrm{TS}^{\ddagger \text { Sulf }}$ respectivamente. Las geometrías de los estados de transición, son presentadas la figura 2 (las líneas punteadas indican ruptura y formación de enlaces). La primera etapa de la reacción muestra la interconversión de la especie azo del colorante rojo allura a la especie hidrazona, pasando por un estado de transición ( $\mathrm{TS}^{\ddagger T a u t}$ ) cíclico de seis miembros, donde se da la transferencia del átomo de hidrógeno desde oxígeno del carbono 2, hacia el átomo de nitrógeno en el grupo azo (figuras 1 y $2 \mathrm{~A}$ ). En cuanto al estado de transición localizado en la segunda etapa (TS ${ }^{\mp \text { Suff }}$ ), el bisulfito se aproxima al carbono en la posición 4, del grupo funcional naftolsulfonato (figuras 1 y $2 \mathrm{~B}$ ), dado su menor impedimento estereoelectrónico frente al resto de átomos de carbono, para generar el respectivo aducto sulfonado.

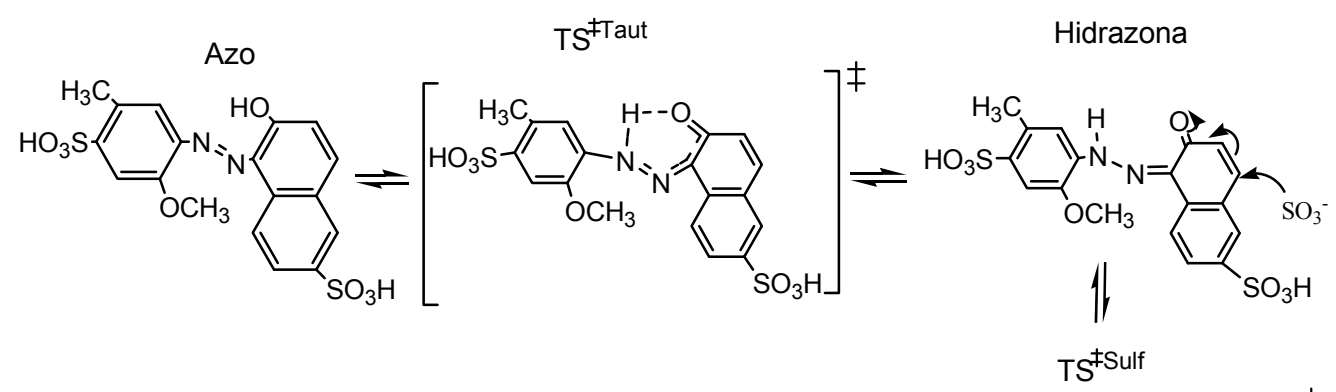

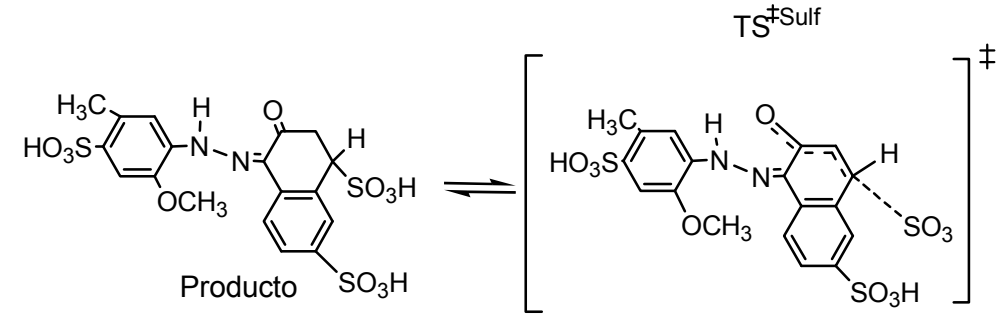

Fig.1: Mecanismo de reacción para la reducción de rojo allura por bisulfito de sodio

En la tabla 1, se presentan las constantes de velocidad $\left(\mathrm{k}_{\text {Taut }}\right)$ y parámetros termodinámicos de activación para el equilibrio azo-hidrazona del colorante rojo allura calculados al nivel B3LYP/6-31G(d) en solución acuosa a diferentes temperaturas. De acuerdo con los datos cinéticos y termodinámicos de la tabla 1, se propone que la reacción de tautomerización transcurre a través de un estado de transición cíclico de seis miembros, concertado y semipolar (figura 2A), ya que la disminución en la entropía de activación (valores 
negativos), sugieren un estado de transición más organizado que los reactivos, con lo que se presume una geometría cíclica para $\mathrm{TS}^{\ddagger T a u t}$. Por otro lado, los valores positivos de la entalpía de activación y energía de Gibbs de activación, indican que la reacción de reducción del colorante es endotérmica, estable, no espontánea o endergónica (Ensuncho et al., 2011; Lafont et al., 2009). Otro aspecto interesante que se puede observar de la tabla 1 , es que los altos valores de $\mathrm{k}_{\text {Taut, }}$ están asociados con valores bajos de $\Delta \mathrm{G}^{*}$, siendo por tanto evidente que el medio juega un papel en la estabilización del estado de transición.

En la tabla 2, se muestran las constantes de velocidad $\left(\mathrm{k}_{\text {sulf }}\right)$ y parámetros termodinámicos de activación, para la segunda etapa de la reacción. Además, se incluyen las constantes de velocidad experimentales $\left(\mathrm{k}_{\mathrm{obs}}\right)$ reportadas por Gemeay (Gemeay, 2002), para el colorante amarillo anaranjado, así como también, los parámetros termodinámicos de activación a $308.15 \mathrm{~K}$. De la tabla 2 , es evidente que el nivel de teoría usado para la simulación fue adecuado, ya que, tanto las constantes de velocidad como los parámetros termodinámicos de activación predichos, estuvieron en buen acuerdo con los datos experimentales reportados para el colorante amarillo anaranjado (Gemeay, 2002). Para evaluar la capacidad del nivel de teoría usado en la predicción de la cinética de reducción experimental determinada para el colorante amarillo anaranjado, (cuya estructura química similar a la de rojo allura), se graficaron el logaritmo natural de la constante de velocidad observada $\left(k_{\text {obs }}\right)$ versus la constante de velocidad predicha $\left(k_{\text {calc }}\right)$. La gráfica generada, se presenta en la figura 3 , donde se puede notar que el nivel de teoría usado es capaz de predecir las constantes de velocidad en buen acuerdo, ya que, se obtuvo un coeficiente de correlación ( $r$ ) de 0.977. Así, $\mathrm{k}_{\mathrm{obs}}$, exhibe una correlación razonable con $\mathrm{k}_{\text {calc }}$, lo cual indica, que el funcional B3LYP en combinación con la base 6-31G(d), es un modelo químico confiable para el estudio de este tipo de sistemas.

Por otro lado, como se esperaba, las constantes de velocidad calculadas en ambas etapas, mantuvieron una relación de proporcionalidad directa con la temperatura, dado que el aumento de la temperatura causa un incremento en la velocidad de reacción.

A

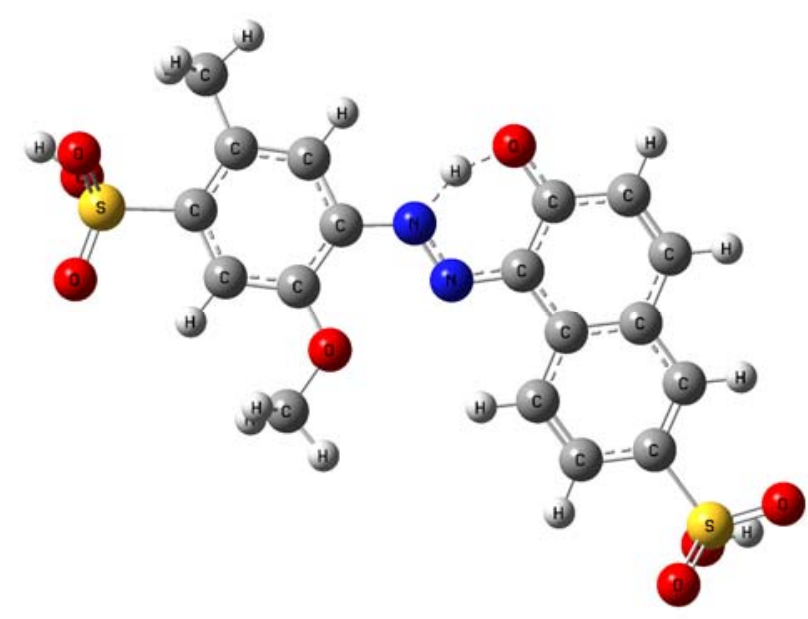

B

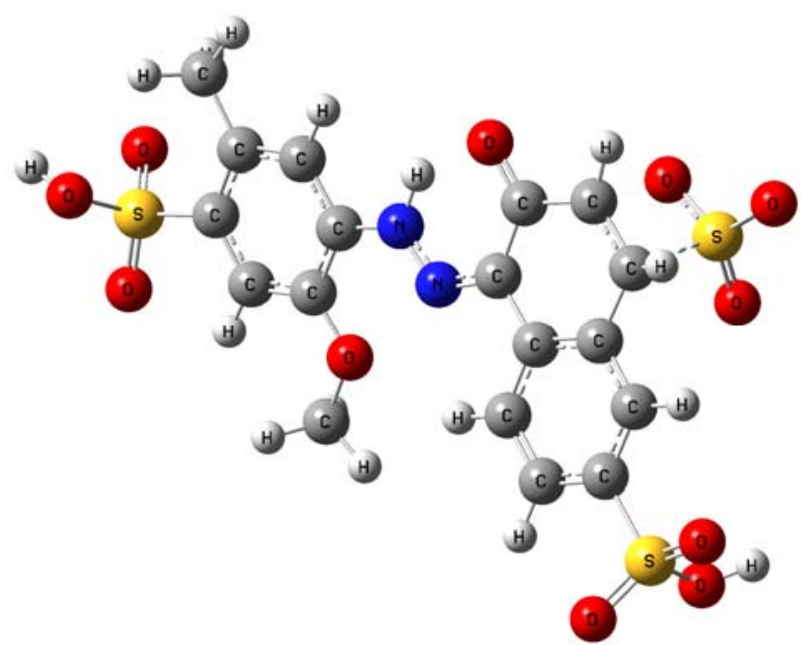

Fig. 2: Geometrías localizadas para los estados de transición de tautomerización (A) y Sulfonación (B) mediante el método QST2

Tabla 1: Constantes de tautomerización y parámetros termodinámicos de activación para el equilibrio azohidrazona del colorante rojo allura calculados en solución acuosa a diferentes temperaturas

\begin{tabular}{|c|c|c|c|c|}
\hline Temperatura $(\mathrm{K})$ & $\mathrm{k}_{\text {Taut }} \times 10^{3} \mathrm{~s}^{-1}$ & $\Delta \mathrm{G}^{\ddagger}(\mathrm{kJ} / \mathrm{mol})$ & $\Delta \mathrm{H}^{\ddagger}(\mathrm{kJ} / \mathrm{mol})$ & $\Delta \mathrm{S}^{\ddagger}(\mathrm{kJ} / \mathrm{mol} . \mathrm{K})$ \\
\hline 298.15 & 7.227 & 50.994 & 47.866 & -0.0104 \\
\hline 303.15 & 9.663 & 51.159 & 47.752 & -0.0112 \\
\hline 308.15 & 12.438 & 51.398 & 47.698 & -0.0120 \\
\hline 313.15 & 15.269 & 51.740 & 47.533 & -0.0134 \\
\hline 318.15 & 20.406 & 51.841 & 47.717 & -0.0130 \\
\hline
\end{tabular}

Para determinar el efecto del solvente sobre la reacción de reducción del colorante, también se determinaron las constantes de velocidad para las dos etapas usando constantes dieléctricas de 2.0 y 6.0 respectivamente. Las constantes de tautomerización predichas, así como los parámetros termodinámicos son presentadas en la tabla 3. Evidentemente, como se muestra en la tabla 3, al disminuir la polaridad del 
solvente, las constantes de tautomerización se incrementan el doble con respecto a la fase acuosa, indicando que en solventes con polaridad baja se favorece la estabilización del estado de transición. De igual manera, para la segunda etapa de la reacción, las ksulf predichas fueron $2.091 \times 10^{-17}(\varepsilon=2.0)$ y 1.32 $\times 10^{-16} \mathrm{~s}^{-1}(\varepsilon=6.0)$ respectivamente. De lo anterior, es claro que en solventes con polaridad baja, la adición del ión bisulfito sobre el colorante rojo allura es significativamente lenta. No obstante, en la fase acuosa se observa que la velocidad de la reacción incrementa, lo cual está en total acuerdo con lo esperado, ya que estas reacciones tienen lugar en matrices liquidas por tratarse de aditivos usados en la industria de alimentos. Ahora bien, es un hecho experimental conocido que el colorante rojo allura, sufre procesos de reducción por acción de la microflora intestinal (Shimada et al., 2010), por lo que el presente estudio incentiva futuros tópicos de investigación en esta área.

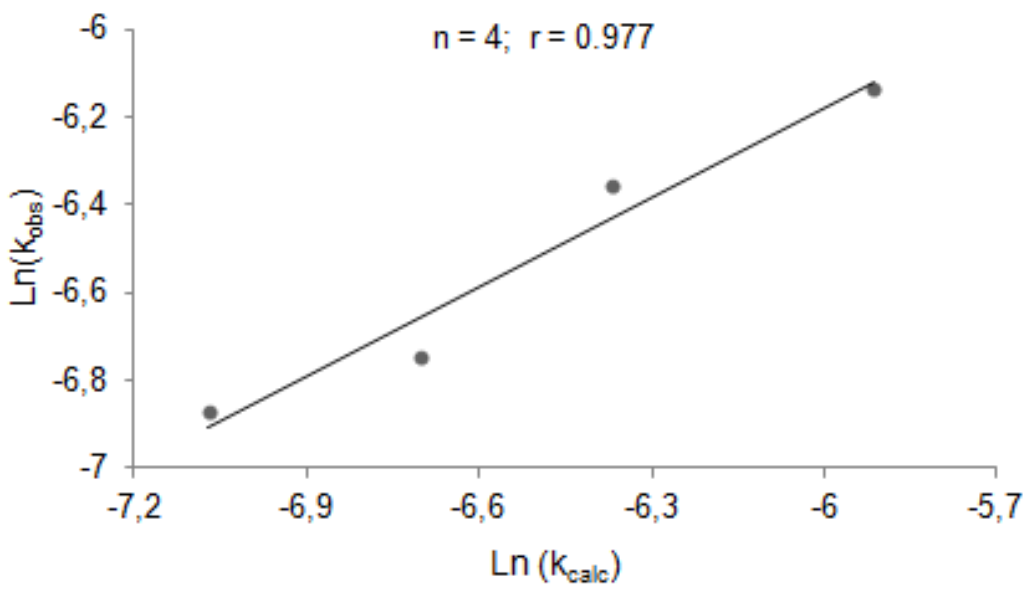

Fig. 3: Gráfico de correlación entre las constantes de velocidad observadas y las predichas para la etapa de sulfonación.

Tabla 2: Constantes de Sulfonación y Parámetros termodinámicos de activación para la reducción de rojo allura predichos a B3LYP/6-31G(d) en fase acuosa

\begin{tabular}{|c|c|c|c|c|}
\hline Temperatura (K) & $\mathrm{k}_{\text {sulf }} \times 10^{-3} \mathrm{~s}^{-1}$ & $\Delta \mathrm{G}^{\ddagger}(\mathrm{kJ} / \mathrm{mol})$ & $\Delta \mathrm{H}^{\ddagger}(\mathrm{kJ} / \mathrm{mol})$ & $\Delta \mathrm{S}^{\ddagger}(\mathrm{kJ} / \mathrm{mol} . \mathrm{K})$ \\
\hline 298.15 & 0.942 & 90.289 & 68.866 & -0.0718 \\
\hline 303.15 & 1.037 & 91.604 & 69.121 & -0.0741 \\
\hline 308.15 & 1.172 & 92.843 & 70.020 & -0.0740 \\
\hline 313.15 & 1.735 & 93.370 & 70.349 & -0.0735 \\
\hline 318.15 & 2.165 & 94.318 & 71.231 & -0.0725 \\
\hline \multicolumn{5}{|c|}{ kobs y parámetros de activación experimentales para amarillo anaranjado } \\
\hline Temperatura (K) & $\mathrm{k}_{\mathrm{obs}} \times 10^{-3} \mathrm{~s}^{-1}$ & $\Delta \mathrm{G}^{\ddagger}(\mathrm{kJ} / \mathrm{mol})$ & $\Delta \mathrm{H}^{\ddagger}(\mathrm{kJ} / \mathrm{mol})$ & $\Delta \mathrm{S}^{\ddagger}(\mathrm{kJ} / \mathrm{mol} . \mathrm{K})$ \\
\hline 298.15 & \multicolumn{2}{|c|}{ No reportado } & --- & --- \\
\hline 303.15 & 0.85 & --- & --- & --- \\
\hline 308.15 & 1.23 & 92.99 & 58.25 & -0.1200 \\
\hline 313.15 & 1.71 & --- & --- & --- \\
\hline 318.15 & 2.70 & --- & --- & --- \\
\hline
\end{tabular}

Tabla 3: Constantes de Tautomerización y Parámetros termodinámicos de activación predichos a 303.15K, usando constantes dieléctricas de 2.0 y 6.0 respectivamente.

\begin{tabular}{|c|c|c|c|c|c|}
\hline Temperatura $(\mathrm{K})$ & $\begin{array}{c}\text { Constante dieléctrica } \\
(\varepsilon)\end{array}$ & $\mathrm{k}_{\text {Taut }} \times 10^{4} \mathrm{~s}^{-1}$ & $\Delta \mathrm{G}^{\ddagger}(\mathrm{kJ} / \mathrm{mol})$ & $\Delta \mathrm{H}^{\ddagger}(\mathrm{kJ} / \mathrm{mol})$ & $\Delta \mathrm{S}^{\ddagger}(\mathrm{kJ} / \mathrm{mol} . \mathrm{K})$ \\
\hline 303.15 & 2.0 & 2.28 & 48.99 & 45.976 & -0.00994 \\
\hline 303.15 & 6.0 & 2.02 & 49.29 & 44.543 & -0.0156 \\
\hline
\end{tabular}




\section{CONCLUSIONES}

Los cálculos computacionales sobre la reacción de reducción del azo colorante rojo allura por bisulfito, fueron cualitativamente consistentes con la evidencia experimental disponible sobre los procesos de degradación del color para azo colorantes de estructura química similar a rojo allura. De acuerdo con las constantes de velocidad, de la reacción de reducción del colorante rojo allura, utilizado en alimentos, dicha reacción transcurre con una velocidad relativamente lenta. Además, para la etapa de tautomerización se propuso por primera vez, un estado de transición cíclico de seis miembros concertado y semipolar, el cual fue compatible con los datos cinéticos y termodinámicos predichos. Se observó que el cambio en la polaridad del solvente afecta las velocidades de reacción en las dos etapas.

\section{AGRADECIMIENTOS}

Al Centro de Investigaciones de la Universidad de Córdoba (CIUC) por financiar el proyecto FCB-07-08 numeral 1120272.

\section{REFERENCIAS}

Berke B, C. Cheze, J. Vercaurteren y G. Deffieux, Bisulfite addition to anthocyanins: revisited structures of colourless adducts, Tetrahedron Lett: 39(32), 5771-5774 (1998).

Beyramabadi, S.A y A. Morsali, Intramolecular proton transfer of 2-[(2,4-dimethylphenyl)iminomethyl]-3,5dimethoxyphenol Schiff-base ligand: A density functional theory (DFT) study, Int. J. Phys. Sci: 6(7), 17801788 (2011).

Del Bene, J.E., y I. Shavitt, Basis-set effects on computed acid-base interaction energies using the Dunning correlation-consistent polarized split-valence basis sets, J. Mol. Struct: 307(0), 27-34 (1994).

Du D., A. Fu y Z. Zhou, Density functional theory study of formamide-formamidic acid tautomerization, Int. J. Quantum. Chem: 99(1), 1-10 (2004).

Damant, A., S. Reynolds y R. Macrae, The structural identification of a secondary dye produced from the reaction between sunset yellow and sodium metabisulphite, Food Addit Contam: 6(3), 273-282 (1989).

Ensuncho, A, N. Milanés y J. R. Robles, Estudio Experimental y Computacional de la Cinética de Termólisis del 2,5-Hexanodiol, Inf. tecnol: 22(6), 87-94 (2011).

Ensuncho, A, J. M. López y J.R. Robles, Reactividad Química de los Azo Colorantes Amarillo Anaranjado y Rojo Allura mediante Descriptores Globales y la Función deFukui, Inf. Tecnol: 23(6), en prensa (2012).

Fallico, B., E. Chiaparra, E. Arena y G. Ballistreri, Assessment of the exposure to Allura Red colour from the consumption of red juice-based and red soft drinks in Italy, Food Addit. Contam. Part A: 28,1501-1515 (2011).

Foresman, J.B. y A. Frisch, Exploring Chemistry With Electronic Structure Methods: A Guide to Using Gaussian, $2^{a}$ edición, 3-302. Gaussian. Inc, USA, Pittsburgh (1996).

Frisch, M., G.W, Trucks y otros 80 autores; Gaussian03, Revision E.01, Gaussian, Inc, Wallingford CT, Pittsburgh, USA (2004).

Gemeay, A.H, Kinetics and mechanism of the reduction of some azo-dyes by inorganic oxysulfur compounds, Dyes Pigments: 54(3), 201-212 (2002).

Holmberg D, Effect of amaranth, Ponceau $4 R$ and/or vitamin $A$ on enzyme activities of the rat liver, Food. Cosmet. Toxicol: 16(1), 1-5 (1978).

Jensen, F, Introduction to computational chemistry, 2a edition, 1-620, John Wiley \& Sons Ltda, England, The Atrium, Southern Gate, Chichester (2006).

Lafont, J, M. Páez y A. Alvis, Estudio cinético de la descomposición térmica de tres derivados del pirano, Infor. Tecnol: 20(5), 39-46 (2009). 
Lee, C., W. Yang y R.G. Parr, Development of the Colle-Salvetti correlation-energy formula into a functional of the electron density, Phys. Rev. B: 37(2), 785-789 (1988).

Levine, I.N., Quantum Chemistry, 6ª Edition, Prentice Hall, India (2008).

Lewars, E.G, Computational Chemistry: Introduction to the Theory and Applications of Molecular and Quantum Mechanics, $2^{\mathrm{a}}$ edition, Springer (2010).

McQuarrie, D.A, Statistical Mechanics, 1a edition, 1-620, Univ Science Books (2000).

Ochterski, J.W., Thermochemistry in Gaussian, Gaussian, Inc., 1-19 (2000).

Özen, A. S., V. Aviyente y R.A. Klein, Modeling the Oxidative Degradation of Azo Dyes: A Density Functional Theory Study, J. Phys. Chem. A: 107(24), 4898-4907 (2003).

Özen, A.S, y V. Aviyente, Modeling the Substituent Effect on the Oxidative Degradation of Azo Dyes, J. Phys. Chem. A: 108(28), 5990-6000 (2004).

Rooney, J.J, Eyring transition-state theory and kinetics in catalysis, J. Mol. Catal. A: Chem., 96(1), L1-L3 (1995).

Ruangpornvisuti, V. y B. Wanno, A DFT investigation of conformational geometries and interconversion equilibria of phenylthiosemicarbazone and its complexation with zinc, J. Mol. Modeling: 10, 418-426 (2004).

Rincón, E. y A. Toro-Labbé, Reaction force and electron localization function analysis of the metal chelation process in Mg(II)-thymine complex, Chem. Phys. Lett: 438(1-3), 93-98 (2007).

Saebo, S y L. Radom, The structure of aminopropenenitrile $\left(\mathrm{CH}_{2} \mathrm{C}\left(\mathrm{NH}_{2}\right) \mathrm{CN}\right)$, J. Mol. Struct: 105(1-2), 119128 (1983).

Salem, M.A. y A.H. Gemeay, Kinetics of the Oxidation of Tartrazine with Peroxydisulfate in the Presence and Absence of Catalyst, Monatsh. Chem: 0117-0129 (2000).

Scotter, M.J. y L. Castle, Chemical interactions between additives in foodstuffs: A review. Food. Addit. Contam: 21, 93-124 (2004).

Shimada, C., y otros cuatro autores, Differential colon DNA damage induced by azo food additives between rats and mice, J. Toxicol. Sci: 35(4), 547-554 (2010).

Tomasi, J., B. Mennucci y R. Cammi., Quantum Mechanical Continuum Solvation Models, Chem. Rev: 105(8), 2999-3094 (2005).

Umer, S., M. Badaruddin, S. Sayeed, R. Ali y M.N. Riaz, Binding ability of Allura Red with food proteins and its impact on protein digestibility, Food. Chem: 110(3), 605-610 (2008).

Wang, Y., X. cheng, X. Yang y X. Yang, DFT Study of Solvent Effects for Some Organic Molecules Using a Polarizable Continuum Model, J. Solution. Chem: 35, 869-878 (2006).

Wilson, B.G. y S.L. Bahna, Adverse reactions to food additives, Ann. Allergy Asthma Immunol: 95, 499-507 (2005).

Yamashita, K., T. Yamabe, y K. Fukui, IRC approach to chemical dynamics: toward mode-selective chemical reactions, Chem. Phys. Lett: 84(1), 123-126 (1981).

Zimmerman, H.E. y P. Wang, Intramolecular Proton Transfer, Org. Lett: 4(15), 2593-2595 (2002). 